\title{
How long will animal markets remain as Borley Rectory?
}

\author{
AbdulRahman Saied ${ }^{1}$ \\ ${ }^{1}$ Affiliation not available
}

July 25, 2020

\begin{abstract}
* SARS and COVID-19; the two deadly pandemics to humans emerged from animal markets * A modern design for animal markets for preventing possible future pandemic diseases coming from animals is a top priority for the current time. * Animal market can be divided into high-risk and low-risk areas. * Visitor policy at the entrance of the market for the safety of animal market visitors.
\end{abstract}

Dear editor,

Wuhan witnessed the emergence of a novel coronavirus (SARS-CoV-2) in late December 2019 which considers a global threat to public health attracting media attention and brings back memories on Spanish Flu pandemic of 1918. SARS-CoV-2 has widely and rapidly spread in China and several other countries, causing an outbreak of acute viral pneumonia.

SARS-CoV-2 posed a high risk on the population level, causing disruptions to the global public health systems and economic losses. Even intense research efforts couldn't define how, when, and where new diseases appear and the relationship that holds whether an outbreak results from a single spillover event (SARS-CoV) or repeated crossing of the species barrier (MERS-CoV) (Munster et al., 2020).

COVID-19 pandemic returns our minds to the ongoing ability of viral spill-over from animals to cause severe disease in humans(Wu et al., 2020). Emerging zoonotic viruses continuously circulate in animal reservoirs and zoonotic spillover making containing pandemic viruses more difficult. It's not new to say that SARS-CoV-2 won't be the last virus transmits in this way.

COVID-19 outbreak originated in Huanan a local Seafood Wholesale market in Wuhan, Hubei province, central China(Wu et al., 2020). In this Sea Market, fish and shellfish, a variety of live wild animals, animal carcasses, and animal meat were available for sale but no bats were available for sale (Wu et al., 2020). Also live-poultry markets have been known to be a source of influenza viruses since the late 1970s(Shortridge, 1992). SARS was isolated from Himalayan palm civets (Pagunalarvata) found in live-animal markets in Guangdong(Guan et al., 2003) .

SARS-CoV-2 sequences are $96 \%$ identical at the whole-genome level to a bat coronavirus detected (Huet al., 2018, Hu et al., 2017, $\mathrm{Xu}$ et al., 2016) that had previously been found in bats in China since 2013(Hu et al., 2018) suggesting that the immediate ancestor of SARS-CoV-2 has been circulating in bats for at least several years. Previous studies have shown that some bat SARSr-CoVs have the potential to infect humans(Menachery et al., 2015, Menachery et al., 2016, Wang et al., 2018) . SARS-CoV-2 is an RNA virus and can mutate (Forniet al., 2017), and consequently SARS-CoV-2 able to adapt in humans providing a more efficient person to person transmission and possibly become more virulent(Wang et al., 2020) .

SARS, MERS, and COVID-9, in all three zoonotic coronavirus pandemics, the ancestral hosts were probably bats. Whether SARS-CoV-2 is transmitted directly from bats (to sellers and market vendors) or through 
intermediate animal hosts (Pangolins (Zhang et al., 2020, Xiao et al., 2020) and other animal species (Sailleau et al., 2020, Deng et al., 2020)) . It's important to understand and define zoonotic transmission patterns.

Cross-species transmission of viruses occurs in some animals sharing territories and water sources with each other and coronaviruses are genetically diverse and undergo frequent mutations and recombination(Zhu et al., 2020, Woo et al., 2012) . So, coronaviruses can jump from animals to humans, from humans to animals and between animals (Graham \& Baric, 2010). Eight million years for the human genome to evolve by $1 \%$ while many animal RNA viruses take days to can evolve by more than $1 \%$ make us expecting the emergence of new zoonotic viruses(Morens et al., 2020) .

Some precautions should be taken in mind for the future:

1. Considerable caution during handling these animals is required.

2. Strict regulations against the domestication and consumption of wildlife should be implemented(Zhou et al., 2020) .

3. Modern design for wet market, live-animal markets.

4. Certified training to who treat, handle, and trade in animals.

5. Vaccination of people with a high risk of exposure to SARS-CoV-2, such as health care workers, veterinarians, slaughterhouse workers, sellers, and market vendors is advisable(Müller et al., 2015)

6. One human cultural practice, in particular, treating and eating wild and domestic animals may save million lives.

7. Sharing experience and learning from all geographical regions and across disciplines.

8. Inappropriate measures taken against domestic or wild animals' trade and consumption owing to COVID-19 may have a negative impact on biodiversity with a view to developing strategies to reduce the risk of future spillover events.

9. Live-animal market protocol: General hygiene measures should be applied, regular hand washing after touching animals and animal products, avoiding contact with stray cats and dogs, rodents, birds, bats, and avoiding contact with animal waste or fluids.

The extensive attention given to animal viruses besides altered human behaviors, environmental changes, and inadequate global public health mechanisms (Allen et al., 2017, Morens et al., 2004, Parrish et al., 2008) could block a future global viral pandemic. We designed an illustration that could be used in a name of "Epidemic Bio-HAACP" to summarize the potential interactions between humans, domestic and wild animals in which probable to occur in our environment, and controlling the possible hazards before their occurrence (Figure 1 ).

SARS and COVID-19; the two deadly pandemics to humans emerged from animal markets and modern design for animal markets for preventing possible future pandemic diseases coming from animals is a top priority for the current time.

Here, we are trying to put a simple ideal design for live animal markets (Figure 2 ) focusing on preventing future pandemic diseases from getting out of the animal world to human community. Closure decision of wet markets isn't easy or practical.

In animal markets; live and slaughtered animals available for sale besides animal products and restaurants for animal-origin foods consumption.

In our Post-COVID-19 design, the main animal market will be divided into two halves. Any entrance must be supplied with a disinfecting channel for market visitors, vendors, and sellers. Vehicles entrances must be provided with disinfecting gates for vendors and sellers vehicles. Precautionary measures as wearing facemask, gloves, and social distancing would be technologically monitored to diminish human contact.

The first one half includes two separate portions for selling of live wild and domestic animals receptively. Each portion has a special entrance with disinfecting channels. The second one half includes two separate 
portions for meat with its different types and its products besides restaurants and a portion for animal products, feed, and accessories respectively.

Market halves connected with long walkaway provided with disinfecting channels. In general, animal markets must be provided by hand washing facilities and hand rub stations present all over the market outlets.

Visitor policy is a protocol for the safety of animal market visitors to begin at the entrance of the market (selling center for face masks and gloves - disinfecting channel) to the exit. Two clinics, a veterinary clinic and medical clinic for emergency cases, and giving advice present in the market.

I think it's important nowadays that every person treats, handle, or trade in animals should be certified to ensure that all persons working in the animal-human interface circle realize how danger of breaking any step in this circle. Also, fostering public education about the risks associated with live animals markets Vaccination of people with a high risk of exposure to emerging disease viruses like SARS-CoV-2, such as health care workers, veterinarians, slaughterhouse workers, sellers, and market vendors is advisable(Müller et al., 2015) . Infrastructure of wet (live animal) markets, visit guidance, and trading guidelines should be crystallized into strict reality.

AcknowledgementsNot applicable

Conflict of InterestThe author declares no conflict of interest relevant to this article.

\section{Ethical Approval}

Not applicable to this comment.Data Availability StatementThere is no experimental data available in this letter.

AbdulRahman A. Saied ${ }^{1,2^{*}}$

${ }^{1}$ Aswan Office, Touristic Activities and Interior Offices Sector, Ministry of Tourism and Antiquities, Aswan 81511, Egypt

${ }^{2}$ Department of Pharmacology, Faculty of Veterinary Medicine, South Valley University, Qena, 83523, Egypt

\section{ORCID}

AbdulRahman A. Saied https://orcid.org/0000-0001-8616-5874

\section{References}

Allen, T., K. A. Murray, C. Zambrana-Torrelio, S. S. Morse, C. Rondinini, M. Di Marco, N. Breit, K. J. Olival and P. Daszak, 2017: Global hotspots and correlates of emerging zoonotic diseases. Nature communications, 8,1-10.

Deng, J., Y. Jin, Y. Liu, J. Sun, L. Hao, J. Bai, T. Huang, D. Lin, Y. Jin and K. Tian, 2020: Serological survey of SARS-CoV-2 for experimental, domestic, companion and wild animals excludes intermediate hosts of 35 different species of animals. Transboundary and Emerging Diseases .

Forni, D., R. Cagliani, M. Clerici and M. Sironi, 2017: Molecular evolution of human coronavirus genomes. Trends in microbiology, 25, 35-48.

Graham, R. L. and R. S. Baric, 2010: Recombination, reservoirs, and the modular spike: mechanisms of coronavirus cross-species transmission. Journal of virology,84, 3134-3146.

Guan, Y., B. Zheng, Y. He, X. Liu, Z. Zhuang, C. Cheung, S. Luo, P. Li, L. Zhang and Y. Guan, 2003: Isolation and characterization of viruses related to the SARS coronavirus from animals in southern China. Science, 302, 276-278.

Hu, B., L.-P. Zeng, X.-L. Yang, X.-Y. Ge, W. Zhang, B. Li, J.-Z. Xie, X.-R. Shen, Y.-Z. Zhang and N. Wang, 2017: Discovery of a rich gene pool of bat SARS-related coronaviruses provides new insights into the origin of SARS coronavirus. PLoS pathogens, 13 . 
Hu, D., C. Zhu, L. Ai, T. He, Y. Wang, F. Ye, L. Yang, C. Ding, X. Zhu and R. Lv, 2018: Genomic characterization and infectivity of a novel SARS-like coronavirus in Chinese bats. Emerging microbes $\mathcal{E}^{3}$ infections, 7, 1-10.

Menachery, V. D., B. L. Yount, A. C. Sims, K. Debbink, S. S. Agnihothram, L. E. Gralinski, R. L. Graham, T. Scobey, J. A. Plante and S. R. Royal, 2016: SARS-like WIV1-CoV poised for human emergence. Proceedings of the National Academy of Sciences, 113, 3048-3053.

Menachery, V. D., B. L. Yount Jr, K. Debbink, S. Agnihothram, L. E. Gralinski, J. A. Plante, R. L. Graham, T. Scobey, X.-Y. Ge and E. F. Donaldson, 2015: A SARS-like cluster of circulating bat coronaviruses shows potential for human emergence.Nature medicine, 21, 1508.

Morens, D. M., P. Daszak and J. K. Taubenberger, 2020: Escaping pandora's box-another novel coronavirus.New England Journal of Medicine .

Morens, D. M., G. K. Folkers and A. S. Fauci, 2004: The challenge of emerging and re-emerging infectious diseases. Nature, 430, 242-249.

Muller, M. A., B. Meyer, V. M. Corman, M. Al-Masri, A. Turkestani, D. Ritz, A. Sieberg, S. Aldabbagh, B.-J. Bosch and E. Lattwein, 2015: Presence of Middle East respiratory syndrome coronavirus antibodies in Saudi Arabia: a nationwide, cross-sectional, serological study. The Lancet Infectious Diseases, 15, 559-564.

Munster, V. J., M. Koopmans, N. van Doremalen, D. van Riel and E. de Wit, 2020: A novel coronavirus emerging in China - key questions for impact assessment. New England Journal of Medicine, 382, 692-694.

Parrish, C. R., E. C. Holmes, D. M. Morens, E.-C. Park, D. S. Burke, C. H. Calisher, C. A. Laughlin, L. J. Saif and P. Daszak, 2008: Cross-species virus transmission and the emergence of new epidemic diseases. Microbiol. Mol. Biol. Rev.,72, 457-470.

Sailleau, C., M. Dumarest, J. Vanhomwegen, M. Delaplace, V. Caro, A. Kwasiborski, V. Hourdel, P. Chevaillier, A. Barbarino and L. Comtet, 2020: First detection and genome sequencing of SARS-CoV-2 in an infected cat in France. Transboundary and emerging diseases .

Shortridge, K., Pandemic influenza: a zoonosis? in Proceedings of the Seminars in respiratory infections, 1992, p. 11.

Wang, C., P. W. Horby, F. G. Hayden and G. F. Gao, 2020: A novel coronavirus outbreak of global health concern. The Lancet, 395, 470-473.

Wang, N., S.-Y. Li, X.-L. Yang, H.-M. Huang, Y.-J. Zhang, H. Guo, C.-M. Luo, M. Miller, G. Zhu and A. A. Chmura, 2018: Serological evidence of bat SARS-related coronavirus infection in humans, China. Virologica Sinica, 33,104-107.

Woo, P. C., S. K. Lau, C. S. Lam, C. C. Lau, A. K. Tsang, J. H. Lau, R. Bai, J. L. Teng, C. C. Tsang and M. Wang, 2012: Discovery of seven novel Mammalian and avian coronaviruses in the genus deltacoronavirus supports bat coronaviruses as the gene source of alphacoronavirus and betacoronavirus and avian coronaviruses as the gene source of gammacoronavirus and deltacoronavirus. Journal of virology, 86, 3995-4008.

Wu, F., S. Zhao, B. Yu, Y.-M. Chen, W. Wang, Z.-G. Song, Y. Hu, Z.-W. Tao, J.-H. Tian and Y.-Y. Pei, 2020: A new coronavirus associated with human respiratory disease in China.Nature, 579, 265-269.

Xiao, K., J. Zhai, Y. Feng, N. Zhou, X. Zhang, J.-J. Zou, N. Li, Y. Guo, X. Li and X. Shen, 2020: Isolation and characterization of 2019-nCoV-like coronavirus from Malayan pangolins. bioRxiv .

Xu, L., F. Zhang, W. Yang, T. Jiang, G. Lu, B. He, X. Li, T. Hu, G. Chen and Y. Feng, 2016: Detection and characterization of diverse alpha-and betacoronaviruses from bats in China. Virologica Sinica, 31, 69-77.

Zhang, T., Q. Wu and Z. Zhang, 2020: Probable pangolin origin of SARS-CoV-2 associated with the COVID19 outbreak. Current Biology . 
Zhou, P., X.-L. Yang, X.-G. Wang, B. Hu, L. Zhang, W. Zhang, H.-R. Si, Y. Zhu, B. Li and C.-L. Huang, 2020: A pneumonia outbreak associated with a new coronavirus of probable bat origin. Nature , 1-4.

Zhu, N., D. Zhang, W. Wang, X. Li, B. Yang, J. Song, X. Zhao, B. Huang, W. Shi and R. Lu, 2020: China Novel Coronavirus Investigating and Research Team. A novel coronavirus from patients with pneumonia in China, 2019. N Engl J Med,382, 727-733.

Animal Market Designs and Biosecurity

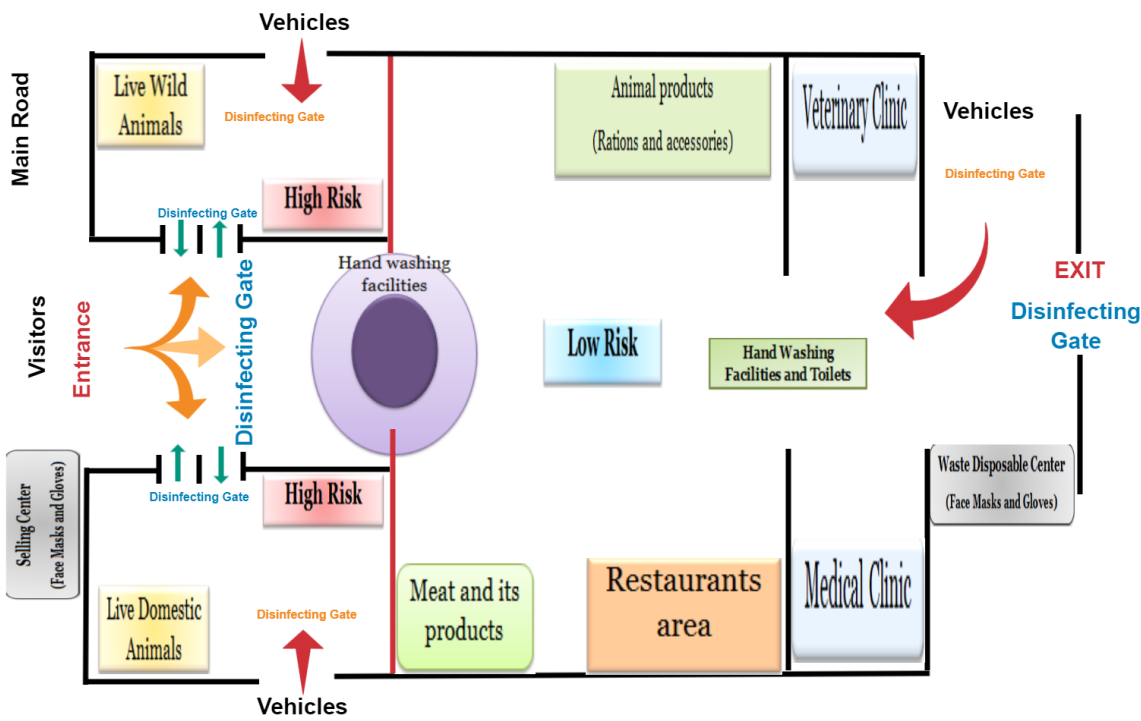


Epidemics Bio-HACCP

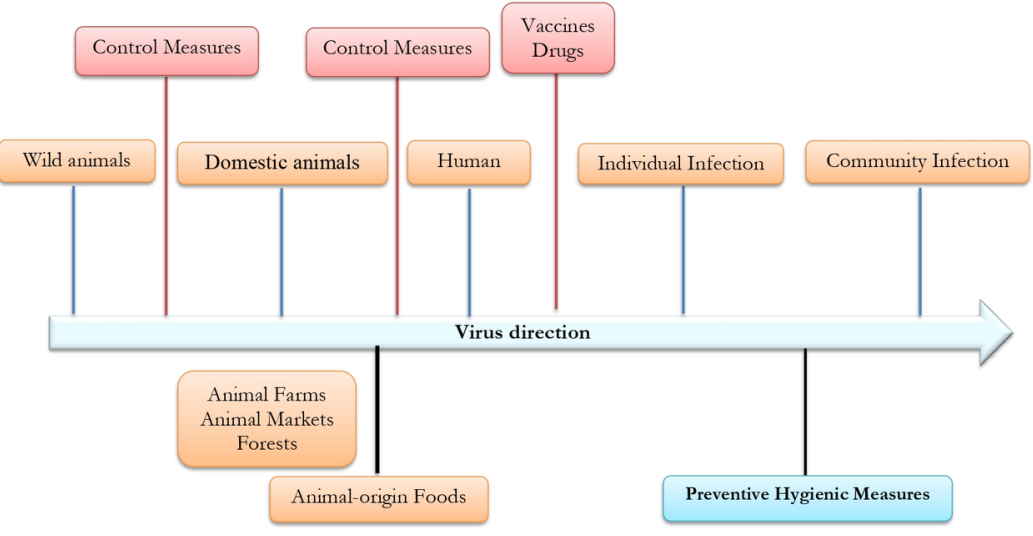

